\title{
Spatial Properties of Network-Mediated Response of Retinal Ganglion Cells to Electric Stimulation*
}

\author{
Maesoon Im and Shelley I. Fried, Member, IEEE
}

\begin{abstract}
Retinal prosthetics consistently demonstrate the ability to restore limited visual perception to those blinded from outer retinal degenerative diseases. However, the performance of retinal implants is highly inconsistent and high-density arrays have proven only marginally better than much sparser ones suggesting that improving the overall quality of elicited vision may require more than just a high density electrode array. Existing devices are also implanted subretinally and epiretinally raising the possibility that electrode location also contributes to percept quality. Here, we compared the responses to stimulation from subretinal and epiretinal electrodes in the same cell. Use of a $4 \times 4$ subretinal electrode array allowed us to also compare responses to different numbers of electrodes activated simultaneously. Surprisingly, responses showed minimal dependence of both the electrode position (epiretinal vs. subretinal) as well as on electrode size (one vs. up to nine electrodes). However, when charge density considerations are implemented, such as those that are necessary during clinical use, the responses arising from smaller electrodes were less effective. This finding may help to explain the inconsistency between theoretical visual acuity and achievement in clinical testing with the high density implanted arrays.
\end{abstract}

\section{INTRODUCTION}

Electric stimulation of surviving retinal neurons reliably elicits visual percepts in subjects that are blind from outer retinal degenerative diseases such as retinitis pigmentosa (RP) and age-related macular degeneration (AMD) [1-6]. Unfortunately, the quality of artificially elicited vision remains fairly limited. To improve clinical outcomes, much effort has focused on developing new implants that contain more electrodes as higher electrode densities are expected to lead to higher spatial resolution [7]. The Alpha-IMS from Retina Implant AG contains 1500 microelectrodes, each $50 \times 50 \mu \mathrm{m}^{2}$ square [3]; this is considerably higher in both count and density than the Argus II from Second Sight, which contains 60 microelectrodes, each $200 \mu \mathrm{m}$ in diameter [8]. Surprisingly however, the Alpha-IMS elicits only marginally better resolution than the Argus II in clinical trials (best visual acuities reported to date are 20/546 [9] and 20/1260 [10], respectively) and the resolutions achieved by the Alpha-IMS do not match its theoretical limit. These results raise questions about the effectiveness of small electrodes during clinical use which is further curious since small electrodes are known to be

* This work was supported by the VA Boston Healthcare System (1I01RX000350-01A1) and by the NIH (1R01EY019967, R01EY023651).

M. Im is with the Massachusetts General Hospital, Harvard Medical School, Boston, MA 02114 USA (e-mail: im.maesoon@mgh.harvard.edu).

S. I. Fried is with the VA Boston Healthcare System, Boston, MA 02130 USA, and the Massachusetts General Hospital, Harvard Medical School, Boston, MA 02114 USA (e-mail: fried.shelley@mgh.harvard.edu, phone: 617-726-3888). more effective than large ones when ganglion cells are directly activated in vitro [11].

In contrast to direct activation of retinal ganglion cells, ganglion cell activity can also arise secondary to activation of the retinal network, e.g. presynaptic bipolar cells or photoreceptors. This mode of activation is referred to as indirect or network-mediated activation. Use of longer duration stimuli, e.g. $>1 \mathrm{~ms}$, is known to preferentially activate through the network [12-14] and has been reportedly used in clinical trials [3] but surprisingly, the effectiveness of small electrodes in activating through the network has not been systematically explored. In addition, while it is well known that network-mediated responses can be initiated from both epiretinal and subretinal positioning of the stimulating electrode, it is not known how the responses vary from the two locations.

Here, we studied how network-mediated responses vary as a function of two parameters. First, we explored the effect of electrode location by comparing responses to epiretinal and subretinal stimulations in the same cell. Second, we investigated how response patterns in the same cell vary as the size of the stimulating electrode is varied. Our results offer some insights for understanding the discrepancy between expected resolution and achieved performance in clinical testing with small-electrode implants.

\section{METHODS}

\section{A. Animal Preparation and Electrophysiology}

Animal handling and surgical procedures followed all federal and institutional guidelines. Protocols were approved by the Institutional Animal Care and Use Committee of the VA Boston Healthcare System or the Subcommittee of Research Animal Care of the Massachusetts General Hospital. After euthanasia, the eyeball was removed; the retina extracted, and then mounted, photoreceptor side down, to a piece of Millipore filter paper that was part of the recording
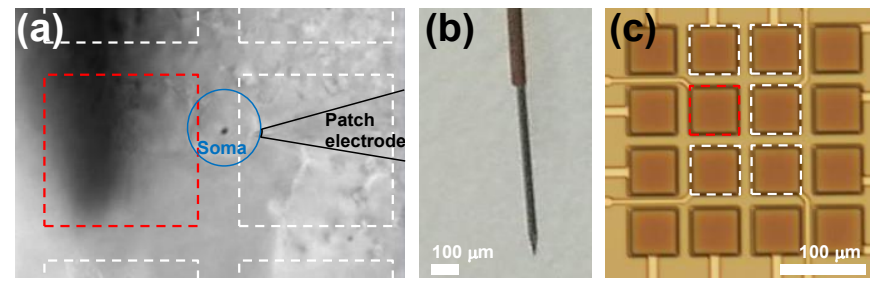

Figure 1. (a) Microscope image of retinal tissue shown with an epiretinal stimulating electrode (dark silhouette on the left) and patch electrode. Soma of targeted ganglion cell is indicated with a circle at the center. Dashed lines indicate positions of individual electrodes within the subretinal array below the retinal tissue. (b) Photograph of the conical epiretinal electrode used in Fig. 1a. (c) Microscope image of the planar subretinal electrode array. The six outlined electrodes outlined with dashed lines were located under the retina tissue near the targeted cell shown are visible in Fig. 1a. 


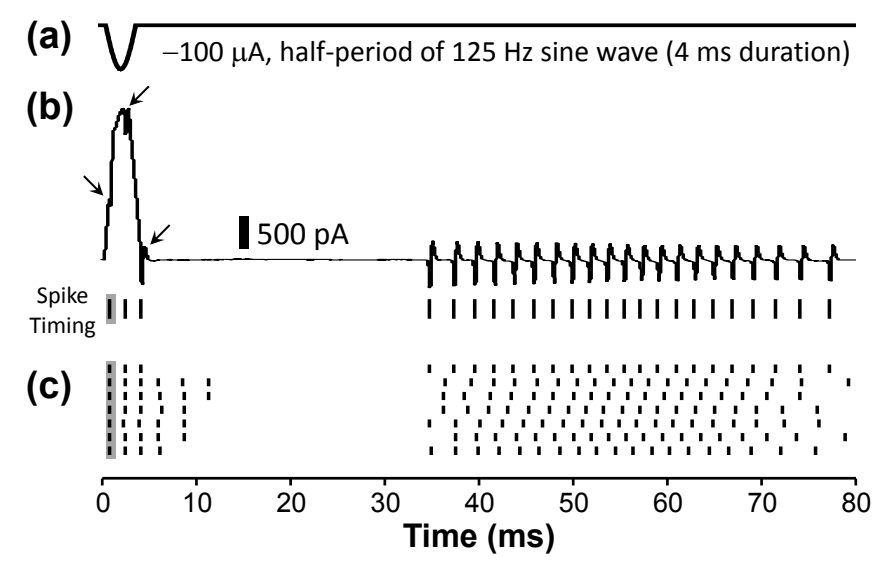

Figure 2. Responses elicited via network-mediated activation. (a) Electric stimulus was a monophasic half-period of $125 \mathrm{~Hz}$ sine wave (4 ms in duration). (b) Raw response to a $-100 \mu \mathrm{A}$ half-sinusoid stimulus. Three spikes are visible within the stimulus artifact (arrows) and many more occur 30-80 ms post-stimulus. (c) Raster responses to 7 presentations (repeats) of the same pulse. The shaded rectangle highlights the first spike - the only one that arises through direct activation of the ganglion cell.

chamber.

Spiking activity of retinal ganglion cell was recorded using a patch pipette (Fig. 1a) in cell-attached mode (4-8 M $\Omega$ ), which was filled with Ames medium. Two $\mathrm{Ag} / \mathrm{AgCl}$ wires served as the reference electrodes and were $\sim 15 \mathrm{~mm}$ away from the targeted cell, positioned at opposite edges of the recording chamber. The retinal sample was maintained at $36^{\circ} \mathrm{C}$ and continuously superfused at $4 \mathrm{~mL} / \mathrm{min}$ with oxygenated Ames medium.

\section{B. Epiretinal Electrode}

The epiretinal electrode used in this study was a $10-\mathrm{k} \Omega$ platinum-iridium electrode (MicroProbes, Gaithersburg, MD) shown in Fig. 1b. The exposed area at the electrode tip was conical with a height of $\sim 125 \mu \mathrm{m}$ and base diameter of $30 \mu \mathrm{m}$, giving a geometric surface area of $\sim 5,900 \mu \mathrm{m}^{2}$ (comparable area to a $\sim 77 \times 77 \mu \mathrm{m}^{2}$ square electrode). The stimulating electrode was positioned $25 \mu \mathrm{m}$ above the inner limiting membrane.

\section{Subretinal Electrode}

A $4 \times 4$ planar electrode array was used for subretinal stimulation. The array was fabricated by Marcus Gingerich at the Cornell Nano-Fabrication Laboratory. Each iridium oxide electrode within the array had an open area of $50 \times 50 \mu \mathrm{m}^{2}$ and center-to-center spacing of $72 \mu \mathrm{m}$, which is similar to the configuration of the Alpha-IMS [3]. Each square electrode could be accessed independently, and multiple electrodes could be activated simultaneously to simulate larger electrodes. For example, we concurrently activated $2 \times 2$ and $3 \times 3$ arrays of adjacent electrodes to simulate be electrode sizes of $100 \times 100$ and $150 \times 150 \mu \mathrm{m}^{2}$, respectively.

\section{Electric Stimulation}

The stimulus waveform was a monophasic current in the form of a half-sinusoidal wave with a duration of $4 \mathrm{~ms}$, corresponding to a half-period of a single $125 \mathrm{~Hz}$ sine wave (Fig. 2a). To parse out effects of the current polarity, stimulus was applied as cathodal- or anodal-only current instead of biphasic waveforms. It is well established that long duration stimuli, both pulsatile and sinusoidal indirectly activate ganglion cells, i.e. ganglion cell activation occurs secondary to activation of presynaptic neurons [12-14]. The $4 \mathrm{~ms}$ duration used here is comparable to the pulsatile waveforms previously shown to generate the strongest retinal responses [15]; it is also comparable to the duration used in clinical trials [3].

Two $\mathrm{Ag} / \mathrm{AgCl}$ wires served as the return for both epiretinal and subretinal stimulation; each wire was positioned $\sim 8 \mathrm{~mm}$ away from the targeted cell and $\sim 6 \mathrm{~mm}$ apart from the other wire. The electric stimuli were applied by a stimulus generator (STG2004, Multi-Channel Systems GmbH, Reutlingen, Germany). The data acquisition and electric stimuli were controlled by custom software written in LabView (National Instruments) and MATLAB (Mathworks, Natick, MA).

\section{E. Data Analysis}

Spiking activity of ganglion cell was sampled at $10 \mathrm{kHz}$ by NI-DAQ (National Instruments, Austin, TX) after low-pass filtering at $2 \mathrm{kHz}$ using an Axopatch 200B amplifier (Molecular Devices, Sunnyvale, CA). Recorded data (Fig. 2b) were further processed offline with customized codes written in MATLAB to detect spikes. Each tick mark shown at the bottom of Fig. $2 b$ represents the timing of elicited spikes detected by post-processing.

\section{RESULTS}

ON brisk transient ganglion cells were exclusively targeted in the present study because of the ease and reliability with which they could be identified, e.g. the presence of doublets in their spontaneous responses unequivocally identifies this type $[16,17]$. Consistent with previous work [15, 18 ], strong cathodal stimuli $(-100 \mu \mathrm{A})$ delivered epiretinally elicited one or more bursts of spikes; the response in Fig. 2b consists of several spikes all with a latency $<\sim 10 \mathrm{~ms}$ followed by a longer burst with an onset latency of $\sim 30 \mathrm{~ms}$. Responses to multiple repeats of the same stimulus were largely consistent in the same cell (Fig. 2c). All but the first spike are consistently eliminated when synaptic input to the ganglion cell is blocked pharmacologically [14, 19-21], suggesting that

(a)

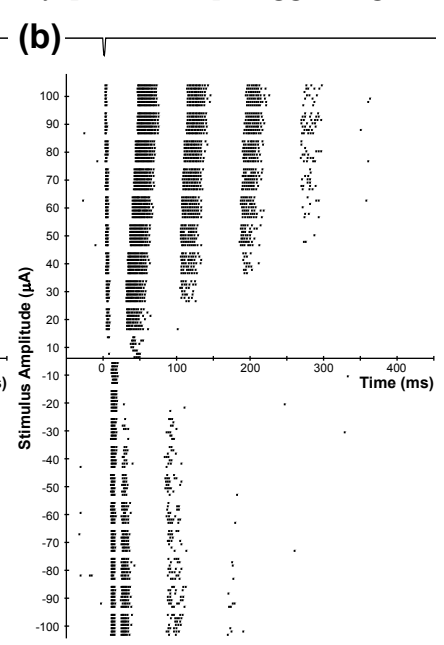

Figure 3. Epiretinal and subretinal stimulations elicit similar response patterns across a wide range of current amplitude. Raster responses from an identical ON BT cell to epiretinal (a) and subretinal (b) stimulations are plotted. Stimulus waveform was same to that of Fig. 2a and amplitudes were changed from -100 to $100 \mu \mathrm{A}$ in $10 \mu \mathrm{A}$ steps. Stimulus was applied at $0 \mathrm{~ms}$ as indicated on top of each panel. 
the first spike (highlighted by shading in Fig. 2c) arises through direct activation of the ganglion cell while all subsequent spikes arise secondary to activation of one or more excitatory presynaptic neurons, e.g. bipolar cells and/or photoreceptors. Thus, the majority of the response arises through network-mediated activation.

\section{A. Comparison of Spiking Patterns in Response to Epiretinal and Subretinal Stimulations}

Fig. 3a shows the response pattern to epiretinal stimuli ranging in amplitude from -100 to $100 \mu \mathrm{A}$ in steps of $10 \mu \mathrm{A}$. Responses to 7 repeats are shown at each level. In order to compare the effect of the location of the stimulating electrode, the same range of stimulus amplitudes was subsequently delivered from a subretinal electrode (positioned underneath the retina - outlined with a red dashed line in Fig. 1a); responses are shown in Fig. 3b. Note that y-axes of the two plots are vertically flipped as the polarity of effective current stimulation is opposite (cathodal and anodal currents for epiretinal and subretinal stimulation, respectively). The two responses patterns are largely similar. For example, the number of bursts elicited at each amplitude is identical. Also, the onset latency of individual bursts increases as a function of stimulus amplitude for later bursts in both cases.

\section{B. Influence of Electrode Size on Network-Mediated Response}

While small electrodes are known to be more effective than large ones for direct activation of ganglion cells [11], their effectiveness for network-mediated activation is not well understood. In order to explore this, we compared responses from three different electrode configurations as illustrated in Fig. 4 (left column). Responses to each configuration are plotted in the corresponding right column (for simplicity, only responses to anodal stimulation are shown). Surprisingly, the patterns were highly similar even though the electrode area in one configuration (Fig. 4c) was nine times greater than that of the smallest configuration (Fig. 4a).

\section{DISCUSSION}

\section{A. Small Differences between Epiretinally and Subretinally Elicited Response Patterns}

The differences in the response patterns arising from network-mediated stimulation were small regardless of whether the electrode was positioned epiretinally or subretinally (Fig. 3). This suggests that the network is activated comparably regardless of which side of the retina stimulation was delivered and is consistent with a previous report from Eickenscheidt et al. [21].

We also found that the electrode geometry (i.e. compare Figs. $1 b$ and 1c) has only a marginal effect on network-mediated responses (Fig. 3). Because the responses arising from a relatively narrow conical electrode are similar to those from large, square electrodes, our results imply that comparable amounts of the retinal network get activated from both configurations. This suggests that the spread of charge from a given electrode is not a limiting factor in network activation. (a) Subretinal electrode $50 \mu \mathrm{m} \times 50 \mu \mathrm{m}$
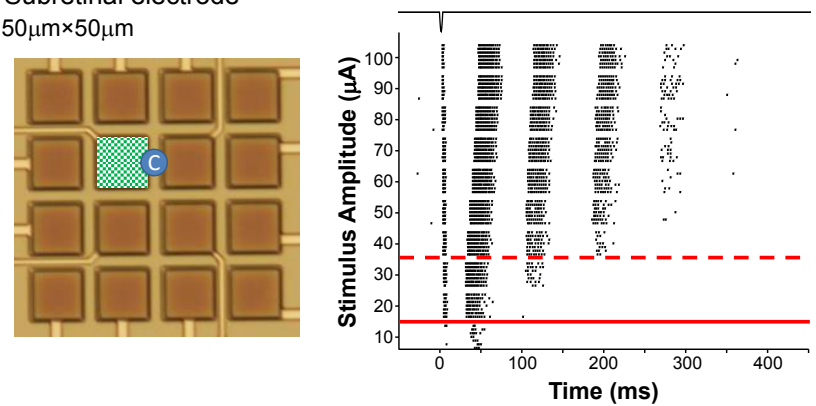

(b) Subretinal electrode $100 \mu \mathrm{m} \times 100 \mu \mathrm{m}$
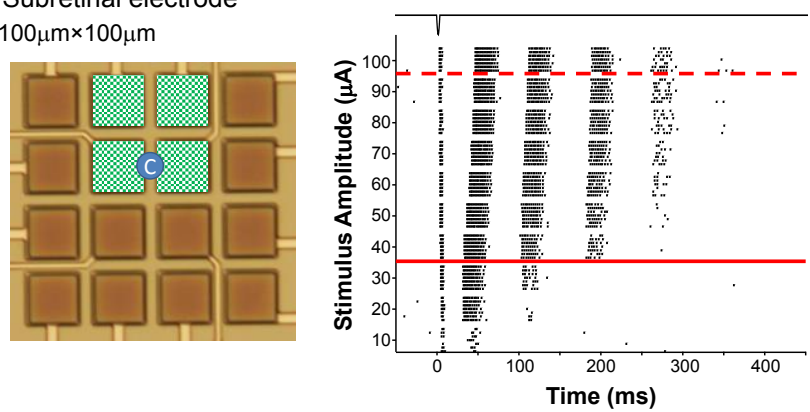

(c) Subretinal electrode $150 \mu \mathrm{m} \times 150 \mu \mathrm{m}$
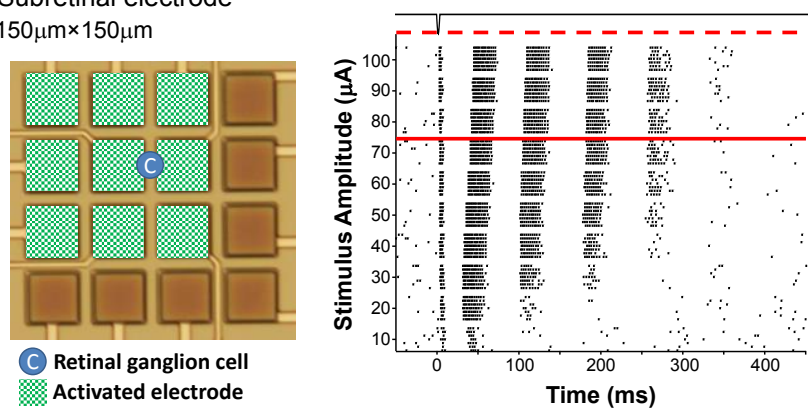

Figure 4. Response patterns are consistent across electrode size and location. Response patterns elicited by a single electrode (A), $2 \times 2$ electrodes corresponding to a $100 \times 100 \mu \mathrm{m}^{2}$ square electrode (B), and $3 \times 3$ electrodes corresponding to a $150 \times 150 \mu \mathrm{m}^{2}$ square electrode. For (B) and (C), the stimulation current indicated on the y-axes was divided equally between all activated electrodes. On each of the three plots, the stimulation amplitudes corresponding to $0.1 \mathrm{mC} / \mathrm{cm}^{2}$ and $0.3 \mathrm{mC} / \mathrm{cm}^{2}$ are indicated with thick solid and dashed lines, respectively. Stimulus was applied at 0 $\mathrm{ms}$ as indicated in the timing trace on top of each raster plot.

\section{B. Charge-density Considerations Impact the Patterns Arising Clinically}

Although the same patterns could be created from different size electrodes, charge-density considerations impact the patterns that can be created safely during clinical use. To prevent adverse chemical reactions from damaging the electrode and/or nearby tissue it is necessary that charge density levels remain below predetermined levels [22], e.g. 0.1 $\mathrm{mC} / \mathrm{cm}^{2}$ is generally considered a 'safe' level for electrodes made out of platinum-iridium. Because the stimulus current was divided equally amongst all activated electrodes, the maximum safe charge that can be delivered to each electrode in the $2 \times 2$ configuration corresponds to $25 \%$ of the stimulus amplitude that gets delivered to the single electrode (charge/electrode was $\sim 11 \%$ for the $3 \times 3$ configuration). As such, the 'safe' charge density level for a fixed amplitude 
stimulus is higher for 'larger' electrodes, e.g. in Fig. 4, the stimulation amplitude corresponding to $0.1 \mathrm{mC} / \mathrm{cm}^{2}$ (indicated by the thick solid line in each plot) is lowest for the single electrode and highest for the $3 \times 3$ configuration. Thus, when operated within safe charge-density limits, e.g. below the red solid line, the $50 \mu \mathrm{m}$ electrode creates weaker patterns of activity while a robust, multi-burst pattern arises in the $3 \times 3$ configuration. Even with a less conservative charge density estimate such as those that might arise for a different electrode material, i.e. $0.3 \mathrm{mC} / \mathrm{cm}^{2}$ (indicated by the thick dashed line in each panel), the patterns elicited at safe levels are more robust for the larger electrodes. This is consistent with an earlier study that suggested that small electrodes were less effective in eliciting percepts than larger electrodes [2]. Our results also suggest that simultaneous activation of multiple nearby electrodes in the Alpha-IMS (or other devices with similar size electrodes) may be necessary in order to elicit robust neural activity.

\section{ACKNOWLEDGMENT}

We thank Seungwoo Lee for technical assistance with experiments that used the subretinal stimulating array, and Marcus Gingerich for fabrication of the subretinal electrode array.

\section{REFERENCES}

[1] M. S. Humayun, E. de Juan Jr, G. Dagnelie, R. J. Greenberg, R. H. Propst, and D. H. Phillips, "Visual perception elicited by electrical stimulation of retina in blind humans," Arch. Ophthalmol., vol. 114, no. 1, pp. 40-46, Jan. 1996.

[2] J. F. Rizzo III, J. Wyatt, J. Loewenstein, S. Kelly, and D. Shire, "Perceptual efficacy of electrical stimulation of human retina with a microelectrode array during short-term surgical trials," Invest. Ophthalmol. Vis. Sci., vol. 44, no. 12, pp.5362-5369, Dec. 2003.

[3] E. Zrenner, K. U. Bartz-Schmidt, H. Benav, D. Besch, A. Bruckmann, V. Gabel, F. Gekeler, U. Greppmaier, A. Harscher, S. Kibbel, J. Koch, A. Kusnyerik, T. Peters, K. Stingl, H. Sachs, A. Stett, P. Szuman, B. Wihelm, and R. Wilke, "Subretinal electronic chips allow blind patients to read letters and combine them to words," Proc. R. Soc. B, vol. 278, no. 1711, pp. 1489-1497, May 2011.

[4] J. D. Weiland, A. K. Cho, and M. S. Humayun, "Retinal prostheses: current clinical results and future needs," Ophthalmology, vol. 118, no. 11, pp. 2227-2237, Nov. 2011.

[5] L. da Cruz, B. F. Coley, J. Dorn, F. Merlini, E. Filley, P. Christopher, F. K. Chen, V. Wuyyuru, J. Sahel, P. Stanga, M. Humayun, R. J. Greenberg, and G. Dagnelie, "The Argus II epiretinal prosthesis system allows letter and word reading and long-term function in patients with profound vision loss," Br. J. Ophthalmol., vol. 97, no. 5, pp. 632-636, May 2013.

[6] M. N. Shivdasani, N. C. Sinclair, P. N. Dimitrov, M. Varsamidis, L. N. Ayton, C. D. Luu, T. Perera, H. J. McDermott, and P. J. Blamey, "Factors affecting perceptual thresholds in a suprachoroidal retinal prosthesis," Invest. Ophthalmol. Vis. Sci., vol. 55, no. 10, pp. 6467-6481, Oct. 2014.

[7] D. K. Freeman, J. F. Rizzo III, and S. I. Fried, "Encoding visual information in retinal ganglion cells with prosthetic stimulation," $J$. Neural Eng., vol. 8, no. 3, pp. 035005, Jun. 2011.

[8] Y. H.-L. Luo, and L. da Cruz, "A review and update on the current status of retinal prostheses (bionic eye)," Br. Med. Bull., vol. 109, no. 1, pp. 31-44, Mar. 2014.

[9] K. Stingl, K. U. Bartz-Schmidt, D. Besch, A. Braun, A. Bruckmann, F. Gekeler, U. Greppmaier, S. Hipp, G. Hörtdörfer, C. Kernstock, A. Koitschev, A. Kusnyerik, H. Sachs, A. Schatz, K. T. Stingl, T. Peters, B. Wilhelm, and E. Zrenner, "Artificial vision with wirelessly powered subretinal electronic implant alpha-IMS," Proc. R. Soc. B, vol. 280, no. 1757, pp. 20130077, Apr. 2013.
[10] M. S. Humayun, J. D. Dorn, L. da Cruz, G. Dagnelie, J.-A. Sahel, P. E. Stanga, A. V. Cideciyan, J. L. Duncan, D. Eliott, E. Filley, A. C. Ho, A. Santos, A. B. Safran, A. Arditi, L. V., Del Priore, and R. J. Greenberg, "Interim results from the international trial of second sight's visual prosthesis," Ophthalmology, vol. 119, no. 4, pp.779-788, Apr. 2012.

[11] C. Sekirnjak, P. Hottowy, A. Sher, W. Dabrowski, A. M. Litke, and E. J. Chichilnisky, "Electrical stimulation of mammalian retinal ganglion cells with multielectrode arrays," J. Neurophysiol., vol. 95, no. 6, pp. 3311-3327, Jun. 2006.

[12] R. J. Greenberg, "Analysis of electrical stimulation of the vertebrate retina - work towards a retinal prosthesis," Ph.D. dissertation, Dept. Biomed. Eng., Johns Hopkins Univ., Baltimore, MD, 1998.

[13] R. J. Jensen, O. R. Ziv, and J. F. Rizzo, "Responses of rabbit retinal ganglion cells to electrical stimulation with an epiretinal electrode," $J$. Neural Eng., vol. 2, no. 1, pp. S16-S21, Mar. 2005.

[14] S. I. Fried, H. A. Hsueh, and F. S. Werblin, "A method for generating precise temporal patterns of retinal spiking using prosthetic stimulation," J. Neurophysiol., vol. 95, no. 2, pp. 970-978, Feb. 2006.

[15] S. W. Lee, D. K. Eddington, and S. I. Fried, "Responses to pulsatile subretinal electric stimulation: effects of amplitude and duration," $J$. Neurophysiol., vol. 109, no. 7, pp.1954-1968, Apr. 2013.

[16] S. H. DeVries, and D. A. Baylor, "Mosaic arrangement of ganglion cell receptive fields in rabbit retina," J. Neurophysiol., vol. 78, no. 4, pp. 2048-2060, Oct. 1997.

[17] E. H. Hu, and S. A. Bloomfield, "Gap junctional coupling underlies the short-latency spike synchrony of retinal $\alpha$ ganglion cells," J. Neurosci., vol. 23, no. 17, pp.6768-6777, Jul. 2003.

[18] D. Tsai, J. W. Morley, G. J. Suaning, and N. H. Lovell, "Direct Activation and Temporal Responses Properties of Rabbit Retinal Ganglion Cells Following Subretinal Stimulation," J. Neurophysiol., vol. 102, no. 5, pp. 2982-2993, Nov. 2009.

[19] A. Stett, W. Barth, S. Weiss, H. Haemmerle, and E. Zrenner, "Electrical multisite stimulation of the isolated chicken retina," Vision Res., vol. 40, no. 13, pp.1785-1795, Jun. 2000.

[20] E. Margalit, and W B. Thoreson, "Inner retinal mechanisms engaged by retinal electrical stimulation," Invest. Ophthalmol. Vis. Sci., vol. 47, no. 6, pp. 2606-2612, Jun. 2006.

[21] M. Eickenscheidt, M. Jenkner, R. Thewes, P. Fromherz, and G. Zeck, "Electrical stimulation of retinal neurons in epiretinal and subretinal configuration using a multicapacitor array," J. Neurophysiol., vol. 107, no. 10, pp.2742-2755, May 2012.

[22] J. O. Winter, S. F. Cogan, and J. F. Rizzo III, "Retinal prostheses: current challenges and future outlook," J. Biomater. Sci. Polym. Ed., vol. 18, no. 8, pp. 1031-1055, Aug. 2007. 